\title{
Night club
}

Growing numbers of amateurs are getting serious about astronomy. The professionals applaud their enthusiasm and success in collecting data and building telescopes - as long as they don't start competing with them for funding. Geoff Brumfiel joins the graveyard shift.

\section{"G} rowing up, I was one of those guys who wanted to be a rock star," says Michael Koppelman. Shortly after he graduated from Boston's Berklee College of Music in 1988, the softspoken, hard-rocking Koppelman began a ten-year pursuit of his dream - working in recording studios with artists such as Prince and Booker T. and the MGs.

But his life took an unexpected turn when his girlfriend gave him a telescope for his birthday a few years ago. "I got the bug real bad," he says. He began by imaging stars and galaxies, but gradually became interested in stars whose brightness changes over time. To monitor such 'variable stars' precisely required advanced equipment and expertise that Koppelman didn't have, so he began cruising Internet chat rooms, talking to others who were interested in doing more than just taking pictures of galaxies and planets. They were a motley bunch from dotcom entrepreneurs to constructionequipment salesmen - whose passion for astronomy drove them to spend thousands of dollars on telescopes and cameras.

Extreme hobbyists can be found in any field, but with the help of cheaper technology, such as digital cameras and powerful personal computers, these amateur astronomers are beginning to take measurements that can rival those of the professionals. And the experts are taking notice: astronomers have begun tapping amateurs for their talent and telescopes, using them to detect asteroids, colliding stars and bursts of high-energy $\gamma$-rays from distant galaxies. Some amateurs are even turning semi-professional, offering their equipment

Garden variety: the amateur-run Tenagra Observatory has provided data for professional stargazers.

and services to full-time researchers for a price (see 'Home help', opposite). "The distinction between amateur and professional astronomers has become very fuzzy," says Arne Henden, a professional astronomer at the US Naval Observatory in Flagstaff, Arizona.

\section{Amateur army}

Across the world, thousands of amateur astronomers point their telescopes skywards each night. Most are sightseers, scanning for colourful clouds of gas or spectacular spiral galaxies, but a few are interested in the more technically challenging aspects of astronomy. They join groups such as the American Association of Variable Star Observers, which since 1911 has enlisted the help of volunteers to monitor variable stars. There are tens of thousands of these stars - too many for professional astronomers to observe regularly - and logging their fluctuations is a full-time operation. Variable stars change brightness for many reasons:

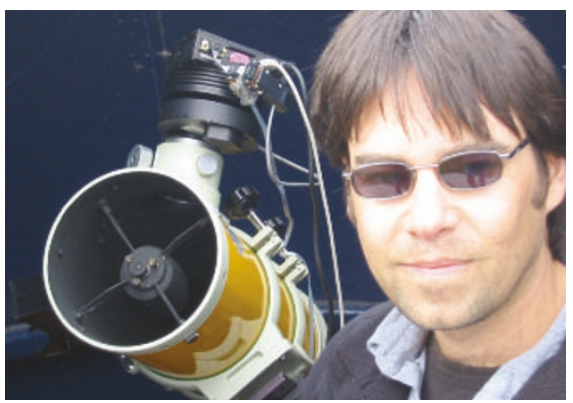

Key change: musician Michael Koppelman wants to swap chords for constellations. they may pulsate or be eclipsed by a companion star, for example. For the brighter stars, all that an association member needs is a telescope and a reference star with which they can visually compare changes in brightness over several nights.

A small fraction of these amateurs take their measurements a step further. They buy advanced digital cameras and computer software that lets them count the light particles coming from the star at any given time. These more detailed measurements are exactly the type of data that Joe Patterson, an astronomer at Columbia University in New York, is looking for. Since the 1980s, Patterson has studied star systems in which a normal star circles around, and is sucked into, a much heavier object such as a black hole. Like variable stars, these star systems brighten and dim over the course of each orbit, but unlike most single stars, the high speed of the orbit means that the changes can take place in a matter of hours. This means that roundthe-clock observations are needed to watch the star as it spins ever closer to its demise.

That's where the amateurs come in, says Patterson. Since the early 1990s he has enlisted the help of enthusiasts around the world to monitor these star systems 24 hours a day. It started with a few well-placed observers on the opposite side of the world from him, but gradually more have signed on, and Patterson's group - the Center for Backyard Astrophysics — now boasts some 40 amateurs from Utah to the Ukraine.

Among the centre's most prolific members is Berto Monard, based in Pretoria, 
